\title{
Combination therapy or monotherapy for the depressed type of schizoaffective disorder
}

\author{
Lubomira Izákovál \\ Ivan Andre' \\ Angelos Halaris ${ }^{2}$ \\ 'Psychiatric Clinic, Faculty \\ of Medicine Comenius University \\ and Faculty Hospital, Bratislava, \\ Slovakia; ${ }^{2}$ Department of Psychiatry \\ and Behavioral Neurosciences, Loyola \\ University Medical Center, Maywood, \\ IL, USA
}

\begin{abstract}
Several studies have demonstrated the effectiveness of adjunctive antidepressant drug therapy to improve the depressive or negative symptoms of schizoaffective disorder, however, monotherapy with atypical antipsychotics may be advantageous. We compared the efficacy and safety of risperidone monotherapy versus combination therapy of haloperidol with sertaline for the acute treatment of schizoaffective disorder, depressed type. This is an open label study of 52 female inpatients randomly assigned to risperidone alone $(\mathrm{N}=26)$ or haloperidol in combination with sertraline $(\mathrm{N}=26)$ for 12 weeks. The mean daily doses of medications were: risperidone: $3.75-3.29 \mathrm{mg}$ /day, haloperidol: $5.35-4.15 \mathrm{mg} /$ day, sertraline: $65.39-133.82 \mathrm{mg} /$ day. Efficacy was measured using clinical rating scales of treatment, safety, and tolerability. Risperidone patients showed statistically significant greater improvement than haloperidol-sertraline patients on efficacy measures including Positive and Negative Syndrome Scale and Clinical Global Impressions rating. A higher number of risperidone patients dropped out of the study early. Fewer adverse events and lesser need for concomitant medications occurred in patients on risperidone. The risperidone group showed better psychological, social and occupational functioning (Global Assessment of Functioning) and higher quality of life (Heinrich's Quality of Life Scale). Risperidone has higher antipsychotic efficacy and tolerability compared with haloperidol-sertraline combination for the acute treatment of schizoaffective disorder, depressed type. Both treatments were comparable in terms of antidepressant efficacy.
\end{abstract}

Keywords: schizoaffective disorder, depressed type, risperidone, haloperidol, sertraline

\section{Introduction}

The existence of schizoaffective disorder as a separate diagnostic entity has been the subject of debate. ${ }^{1-3}$ It has often been considered to be the "waste" basket of a diagnosis by exclusion. Nevertheless, the combination of schizophrenic and depressive symptoms represents a clinical reality requiring accurate diagnosis and effective management. The prognosis of schizoaffective disorder is usually more favorable than for schizophrenia, but less positive in comparison to affective disorders. ${ }^{4}$ The depressed type of schizoaffective disorder represents a challenge due to the chronic and frequently deteriorating course of the illness with negative effects on all spheres of social functioning. The net result is higher utilization of health care resources. 5,6 A factor that can significantly influence the prognosis for this disorder is the choice of suitable therapeutic strategy, in the acute, as well as the continuation and maintenance phases of treatment.

The pharmacotherapy of the acute phase of schizoaffective disorder involves primarily antipsychotic and secondarily antidepressant drugs. However, to achieve an optimal effect, patients with schizoaffective disorder, depressed type, require targeted pharmacologic therapy aimed at improving the schizophrenic as well as the affective components of the illness. Studies focusing on the pharmacotherapy of schizoaffective disorder, depressed type, are limited. Most studies have focused on 
the efficacy and safety of antipsychotic monotherapy while opinions regarding the appropriateness of combined antipsychotic-antidepressant therapy vary. Antidepressants are especially useful when antipsychotic treatment improves the psychotic symptoms only. ${ }^{7}$

Due to their efficacy and safety profile atypical antipsychotics should result in improved long-term efficacy, quality of life, and patient compliance. Karow and colleagues ${ }^{8}$ found a subjective preference for atypical antipsychotics by patients with schizophrenia and schizoaffective disorder, who had received long-term antipsychotic medication. As has been documented by Clark and colleagues, ${ }^{9}$ the use of atypical antipsychotics in patients with schizophrenia and schizoaffective disorder increased from $43 \%$ to $70 \%$ between 1995 and 1999. However, a surprising finding of this study was that, in the era of new-generation antipsychotics with broad-spectrum efficacy, combination antipsychotic therapy increased from $5.7 \%$ to $24.3 \%$. In their meta-analysis of trials aimed at comparing long-term treatment with typical and atypical antipsychotics in patients with schizophrenia and schizoaffective disorder published between 1994 and 2005, Turner and Stewart ${ }^{10}$ observed a lower number of relapses, fewer adverse events (AEs) and higher efficacy of atypical antipsychotics. Flynn and colleagues ${ }^{11}$ retrospectively studied therapy in 70 hospitalized patients with schizoaffective disorder (depressive symptoms present in 53\% patients): $90 \%$ of patients received antipsychotics while the proportion of atypical antipsychotics increased year-to-year; 87\% patients received an antipsychotic drug in combination with an antidepressant or mood stabilizer; only $6 \%$ of patients received monotherapy with a typical or atypical antipsychotic drug. There was a tendency to continue the antidepressant if the patient was receiving such treatment before hospitalization despite not currently experiencing depressive symptoms. The presence of depressive symptoms at the time of hospitalization rarely leads to the administration of an antidepressant. Flynn and colleagues did not confirm that monotherapy with atypical antipsychotics is a widespread method in clinical practice.

We had earlier conducted a retrospective pilot study to obtain an overview of current treatment strategies in the acute phase of treatment of schizoaffective disorder, depressed type. ${ }^{12}$ We determined that the most prevalent pharmacotherapeutic strategy was the combination of antipsychotics with antidepressants. The reasons for preferring such combined treatment over monotherapy were: (a) low incidence of AEs with selective serotonin reuptake inhibitors (SSRIs) and serotonin-norepinephrine reuptake inhibitors (SNRIs), (b) statistically significant effect on reduction of depressed symptoms, (c) decreased risk of relapse when changing the antipsychotic medication. The treatment of schizoaffective disorder in Slovakia was also studied by Dóci and colleagues ${ }^{13}$ In this study, the combination of antipsychotics with antidepressants also prevailed over monotherapy with an antipsychotic drug, while atypical antipsychotics were preferred.

The present 12-week study was designed to compare the efficacy and safety of monotherapy with risperidone versus combination therapy with haloperidol and sertraline in the acute phase of schizoaffective disorder, depressed type.

\section{Methods and materials Study design}

Open label, randomized, prospective, clinical study.

\section{Inclusion and exclusion criteria}

The study was meant to include men and women, aged 18-65, hospitalized for schizoaffective disorder, depressed type, diagnosed by ICD-10 criteria (The International Classification of Diseases, 10th Revision) and DSM-IV (Diagnostic and Statistical Manual of Mental Disorders, 4th Edition). The nature of the episode was documented as first or recurring episode, relapse or gradual decompensation. The negative symptoms subscore had to be higher than the positive symptoms subscore (Positive and Negative Syndrome Scale [PANSS]) at the initial assessment. The total score on the Calgary Depression Scale for Schizophrenia (CDSS) had to be $\geq 5$ at the initial assessment. Patients previously receiving a depot antipsychotic were included in the study only after a minimum of one therapeutic interval had lapsed. Women of reproductive age had to be practicing reliable contraception. The study was approved by the Institutional Ethics Committee and all patients had to give written informed consent before enrolling in the study. Exclusion criteria included the presence of a mental disorder other than that specified in the inclusion criteria, the need for electroconvulsive therapy, and a history of insufficient efficacy of the study medication. Patients with a serious or unstable medical condition were excluded; however, patients with a chronic illness who were stable on medication were allowed into the study.

\section{Treatment}

\section{Risperidone monotherapy (Group R)}

Risperidone was begun at $1 \mathrm{mg} /$ day and subsequently titrated according to the condition of the patient. The mean daily dose of risperidone at week 1 was $3.75 \mathrm{mg} /$ day $(\mathrm{SD}=1.37)$ and at the end of week 12 it was $3.29 \mathrm{mg} /$ day $(\mathrm{SD}=1.51)$. 


\section{Combined haloperidol and sertraline therapy (Group HS)}

The initial dose of haloperidol was determined on the basis of the patient's clinical condition. The initial dose of sertraline was $50 \mathrm{mg} /$ day. The doses of combined therapy were individually and flexibly adjusted. A low dose of haloperidol was preferred throughout the entire study. The mean daily dose of haloperidol at week 1 was $5.35 \mathrm{mg} /$ day $(\mathrm{SD}=3.97)$ and that of sertraline was $65.39 \mathrm{mg} /$ day $(\mathrm{SD}=23.53)$. At week 12 the mean daily dose of haloperidol was $4.15 \mathrm{mg}$ /day $(\mathrm{SD}=3.38)$ and the dose of sertraline was $133.82 \mathrm{mg} /$ day $(\mathrm{SD}=38.47)$. Anticholinergics, benzodiazepines, and nonbenzodiazepine hypnotics were allowed at the discretion of the physician and were recorded in the patient's chart.

After obtaining informed consent, the patient's eligibility for inclusion in the study was assessed. The patient then underwent an initial examination and was assigned a randomized identification code. A detailed record of previous psychotropic and nonpsychotropic medications was obtained. All psychotropic medications were discontinued and the study medication was initiated.

Assessment of psychopathology and efficacy and safety of study medication were recorded at weeks 1,2 , and subsequently at two-week intervals. The final assessment occurred after 12 weeks, or in case of an early drop-out, at the time of termination. All data were recorded in the patient's chart. In case of an early drop-out, the reason was recorded as well.

Instruments for assessment of therapeutic efficacy

- Positive and Negative Syndromes Scale for Schizophrenia (PANSS) ${ }^{14}$

- Calgary Depression Scale for Schizophrenia (CDSS) $)^{15,16}$

- Clinical Global Impression (CGI) (CGI-S; Severity of Illness, CGI-I; Improvement) ${ }^{17}$

- Heinrich's Quality of Life Scale (HQLS) ${ }^{18}$

- Global Assessment of Functioning Scale (GAF) ${ }^{19}$

- Drug Attitude Inventory (DAI) ${ }^{20}$

- Patient Preference Scale (PPS) ${ }^{21}$

Instruments for assessment of treatment safety

- Adverse events report (AER)

- Simpson-Angus Extrapyramidal Symptoms Rating Scale (SAS) ${ }^{22}$

- Laboratory tests, vital signs, body weight

\section{Statistical analyses}

The demographic and clinical variables are described based on characteristics of the study groups R and HS, and reciprocally compared using the Mann-Whitney U test and chi-square test. The characteristics of both groups were assessed separately for the baseline (week 0), end of study (week 12), and for the drop-out groups. The collected data represent quantifiable differences of grouped median scores of the assessment scales in respective assessed time points, which were analyzed using the method of "Observed Cases." As the sets of patients are number-restricted, the statistical assessment applied nonparametric tests. For assessing statistical significance, we applied the significance level of at least $5 \%(\mathrm{p}=0.05)$. We placed emphasis on effect size, calculated as the correlation rate of effect size of difference between grouped medians (correlation rate $\mathrm{r}_{\mathrm{m}}$ ). The correlation rate (effect size) is standardized, and reaches values from 0 to 1 . Its size will be interpreted as: $0.00-0.30$ small, $0.30-0.50$ medium, over 0.50 large.

In the statistical evaluation of variance in scores and differences of scores in individual weeks in scales PANSS, CDSS, CGI-I, CGI-S, PPS, HQLS, DAI within each group, the Wilcoxon test $(z)$ and the correlation rate of effect size between grouped medians $\left(\mathrm{r}_{\mathrm{m}}\right)$ were applied. The statistical evaluation of differences between individual groups and statistical evaluation of score variances in individual weeks used the Mann-Whitney U test and correlation rate of effect size between grouped medians $\left(\mathrm{r}_{\mathrm{m}}\right)$. The two-way significance level (p) was evaluated. The interscale correlations within both groups and between the groups were assessed using correlation analysis. The primary indicator for evaluating the tightness of relation of two quantitative variables was determining the Spearman rank correlation coefficient rho. The statistical software SPSS (SPSS Inc., Chicago, IL) and Excel 2000 (Microsoft Corp., Redmond, WA) were used for all analyses.

\section{Results}

\section{Demographic and clinical characteristics of patients}

Two thousand four hundred sixty-six patients were screened for the study. At the time of discharge from the hospital 207 patients (32.9\% male, $67.1 \%$ female) were diagnosed with schizoaffective disorder. Of these, 130 patients $(62.8 \%)$ were treated for schizoaffective disorder, depressed type, and 77 patients (37.2\%) for manic type. None of the cases had the mixed type of schizoaffective disorder. The sex distribution of patients with schizoaffective disorder, depressed type, was almost $4: 1$ in favour of females $(78.5 \%$ female vs $21.5 \%$ male) (Table 1).

When patient enrolment into the study was completed, 54 patients ( 52 female) had met inclusion criteria. The only 
two male patients who met inclusion criteria dropped out at week 4, due to noncompliance with the study protocol. They were not included in the statistical analysis. The subject population included in the statistical analysis consisted of 52 female patients, representing $2.1 \%$ of the initial group. The study patients were randomly assigned in a 1:1 ratio, either to the risperidone monotherapy (Group R) or to the haloperidol-sertraline combination therapy (Group HS). The randomization codes were assigned using the method of random number selection. The total duration of the study was 12 weeks. All patients were hospitalized at the beginning of the study, and all were followed as outpatients after discharge from the inpatient service. Two patients were enrolled in the study at two different hospitalizations, after suffering a relapse three years later. A solid remission in both of these patients was obtained between hospitalizations. Since both patients were randomized at their second hospitalization and turned out to have been assigned to the other treatment group, they were kept in the data analysis.

Demographic and clinical characteristics of Groups $\mathrm{R}$ and HS at baseline are listed in Table 2. There were 14 dropouts in Group R and 9 dropouts in Group HS. There were no statistically significant differences in demographic and clinical characteristics at weeks 0 and 12 (Mann-Whitney $\mathrm{U}$ test, chi-square test). Groups R and HS differed at week 0 in living arrangement $\left(r_{m}=0.47\right)$ and at week 12 in level of education $\left(r_{m}=0.30\right)$ (Table 2$)$.

\section{Evaluation of therapeutic efficacy \\ Positive and Negative Syndromes Scale for Schizophrenia}

The subscore of positive and negative symptoms, the subscore of general psychopathology and the total PANSS score

Table I Population of patients with diagnosis F25.x according to ICD- 10

\begin{tabular}{ll}
\hline $\begin{array}{l}\text { Population of inpatients } \\
\text { in years 2003-2006 (n) }\end{array}$ & $\begin{array}{l}\text { Number } \\
\mathbf{2 4 6 6}\end{array}$ \\
\hline with diagnosis F25.x (n/\%) & $207 / 8.4 \%$ \\
F25.0 manic type $(\mathrm{n} / \%)$ & $77 / 37.2 \%$ \\
Male & $40 / 51.9 \%$ \\
Female & $37 / 48.1$ \\
F25.I depressed type $(\mathrm{n} / \%)$ & $130 / 62.8 \%$ \\
Male & $28 / 21.5 \%$ \\
Female & $102 / 78.5 \%$ \\
F25.2 mixed type $(\mathrm{n} / \%)$ & $0 / 0.0 \%$ \\
Male & $0 / 0.0 \%$ \\
Female & $0 / 0.0 \%$ \\
\hline
\end{tabular}

in Groups R and HS improved significantly from week 0 to week 12. In comparing Groups R and HS at week 0, a statistical significance $(\mathrm{p}=0.004)$ and medium effect size $\left(r_{m}=0.39\right)$ was obtained only in the subscore of positive symptoms in favour of group R. At weeks 2 and 6, no statistically significant differences in the total score and the PANSS subscores were obtained between the two Groups. At week 6 , the effect size of the total PANSS score $\left(r_{m}=0.30\right)$ and the subscore of general psychopathology $\left(r_{m}=0.32\right)$ suggest a significant difference in favour of Group R. These differences gain statistical significance and a large effect size at week $12\left(\mathrm{r}_{\mathrm{m}}=0.47 ; \mathrm{r}_{\mathrm{m}}=0.56\right)$ (Figure 1).

\section{Calgary Depression Scale for Schizophrenia}

Improvement in depressive symptoms is demonstrated by the reduction in CDSS scores between weeks 0 and 12 in both groups. Group HS retained a higher CDSS score throughout the entire study. At the beginning of the study, the Groups differed significantly $(\mathrm{p}=0.001)$, reflecting an effect of randomization, and the effect size was medium $\left(r_{m}=0.46\right)$. To control for the significant difference in baseline scores between the two groups, we also compared scores in weeks $0-2,2-6$ and 6-12. During the course of the study the difference between Groups diminished slightly. At week 6, the difference was no longer statistically significant $(\mathrm{p}=0.136$; $\left.r_{m}=0.23\right)$, however, it slightly increased again at week $12\left(\mathrm{p}=0.047 ; \mathrm{r}_{\mathrm{m}}=0.32\right)$ (Figure 2$)$.

\section{Clinical Global Impression}

The severity of illness, as assessed by the CGI-severity (CGI-S) scale, decreased significantly $(\mathrm{p}=0.01)$ in both groups with a large effect size $\left(r_{m}>0.60\right)$. The severity of illness was comparable in both groups at the beginning (grouped medians: Group R: 5.06; Group HS: 5.46 points). At week 6 , a statistically significant difference $(p=0.048)$ and a medium effect size $\left(r_{m}=0.32\right)$ in favor of Group $R$ emerged, and from the perspective of effect size it persisted until week $12\left(\mathrm{r}_{\mathrm{m}}=0.33\right)$. Clinical improvement assessed by CGI-I was statistically significant with a large effect size in the course of the study in both groups. Improvement was more rapid during the first six weeks.

\section{Heinrich's Quality of Life Scale}

Both groups showed improvement in the quality of life (grouped medians: Group R: 23.44 vs 34.67 points; Group HS: 22.20 vs 31.33 points). There was no statistically significant difference and no effect size at the beginning of the study, however, Group HS achieved lower HQLS scores throughout the study. At the end of week 12, a medium 




Figure I The grouped medians of Positive and Negative Syndrome Scale total scores at weeks 0, 2, 6, 12 .

Notes: R Group R; HS Group HS; ${ }^{p} \mathrm{p} \leq 0.05 ;{ }^{* *} \mathrm{p} \leq 0.001 ;{ }^{\dagger} r_{\mathrm{m}} \geq 0.3 ;{ }^{\ddagger} \mathrm{r}_{\mathrm{m}} \geq 0.5$. Wilcoxon test $(\mathrm{z})$ and effect size $\left(r_{\mathrm{m}}\right)$ : Group R: week 0-2:z=-4.281;p<0.001; $r_{\mathrm{m}}=0.65 ;$ week $0-6: z=-3.724 ; p<0.001 ; r_{m}=0.67$; week $0-12: z=-3.062 ; p<0.001 ; r_{m}=0.68$. Group HS: week $0-2: z=-4.398 ; p<0.001 ; r_{m}=0.66 ;$ week $0-6: z=-4.019 ; p<0.001$; $r_{m}=0.67$; week $0-12: z=-3.622 ; p<0.001 ; r_{m}=0.67$. Mann-Whitney $U$ test $(Z)$ and effect size $\left(r_{m}\right)$ : Group R vs Group HS; week 0:320.50; $Z=-0.320 ; p=0.754 ; r_{m}=0.04$; week 2: $317.50 ; Z=-0.375 ; p=713 ; r_{m}=0,05 ;$ week $6: 123.00 ; Z=-1.861 ; p=0.063 ; r_{m}=0.30$; week $12: 46.50 ; Z=-2.465 ; p=0.012 ; r_{m}=0.47$.

effect size between Groups favoured Group $\mathrm{R}(\mathrm{r}=0.30)$ (Figure 3).

\section{Global Assessment of Functioning Scale}

Overall functioning in both groups improved significantly during the study $\left(\mathrm{p}<0.001 ; \mathrm{r}_{\mathrm{m}}>0.60\right)$. At week 0 , groups did not differ significantly and there was no effect size. The difference between groups grew during the study.
At week 12 the difference became statistically significant and there was a medium effect size in favor of Group $\mathrm{R}$ $\left(\mathrm{p}=0.044 ; \mathrm{r}_{\mathrm{m}}=0.38\right)$ (Figure 4).

\section{Drug Attitude Inventory}

Patients' attitude towards therapy showed a positive change during the study. In Group R, we noted an increase in DAI score from -4.29 at week 1 to 6.67 points at week 12 .

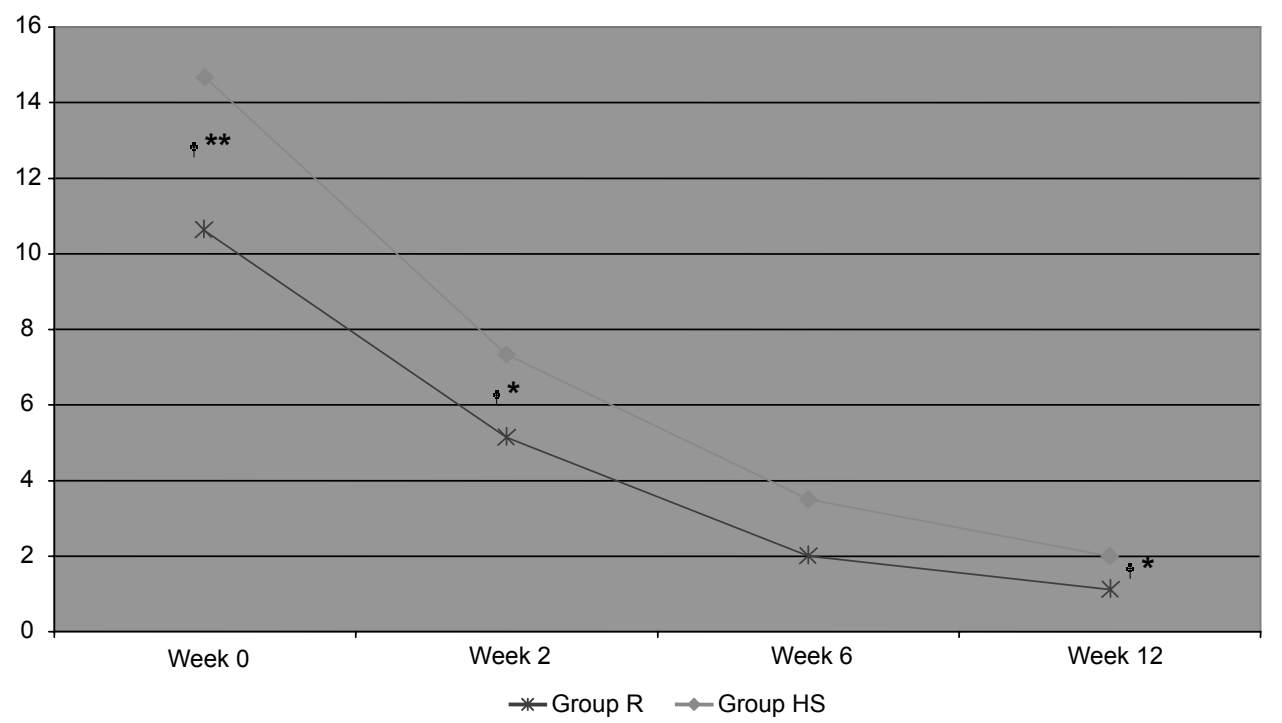

Figure 2 The grouped medians of Calgary Depression Scale for Schizophrenia score at weeks 0, 2, 6, 12.

Notes: *p $\leq 0.05 ;{ }^{* *} \mathrm{p} \leq 0.00 \mathrm{I} ;{ }^{\dagger} \mathrm{r}_{\mathrm{m}} \geq 0.3$. Wilcoxon test $(\mathrm{z})$ and effect size $\left(\mathrm{r}_{\mathrm{m}}\right)$ : Group R: week $0-2: \mathrm{z}=-3.868 ; \mathrm{p}<0.00 \mathrm{I} ; \mathrm{r}_{\mathrm{m}}=0.6 \mathrm{I} ;$ week $0-6$ : $\mathrm{z}=-3.969 ; \mathrm{p}<0.00 \mathrm{I}$; $r_{m}=0.66$; week 0-12: $z=-3.070 ; p<0.00 I ; r_{m}=0.68$. Group HS: week $0-2: z=-4.295 ; p<0.00 I ; r_{m}=0.66 ;$ week $0-6: z=-4.021 ; p<0.001 ; r_{m}=0.67 ; t$ week $0-12$ : $z=-3.626 ; p<0.00 I ; r_{m}=0.67$. Mann-Whitney $U$ test $(Z)$ and effect size $\left(r_{m}\right)$ : Group R vs Group HS: week 0: I58.50; $Z=-3.303 ; p=0.00 I ; r_{m}=0.46 ;$ week 2: 207.50; $Z=-2.228 ; p=0.025 ; r_{m}=0.32 ;$ week $6: 162.00 ; Z=-1.502 ; p=0.136 ; r_{m}=0.23 ;$ week I2:59.50; $Z=-2.502 ; p=0.047 ; r_{m}=0.32$. 


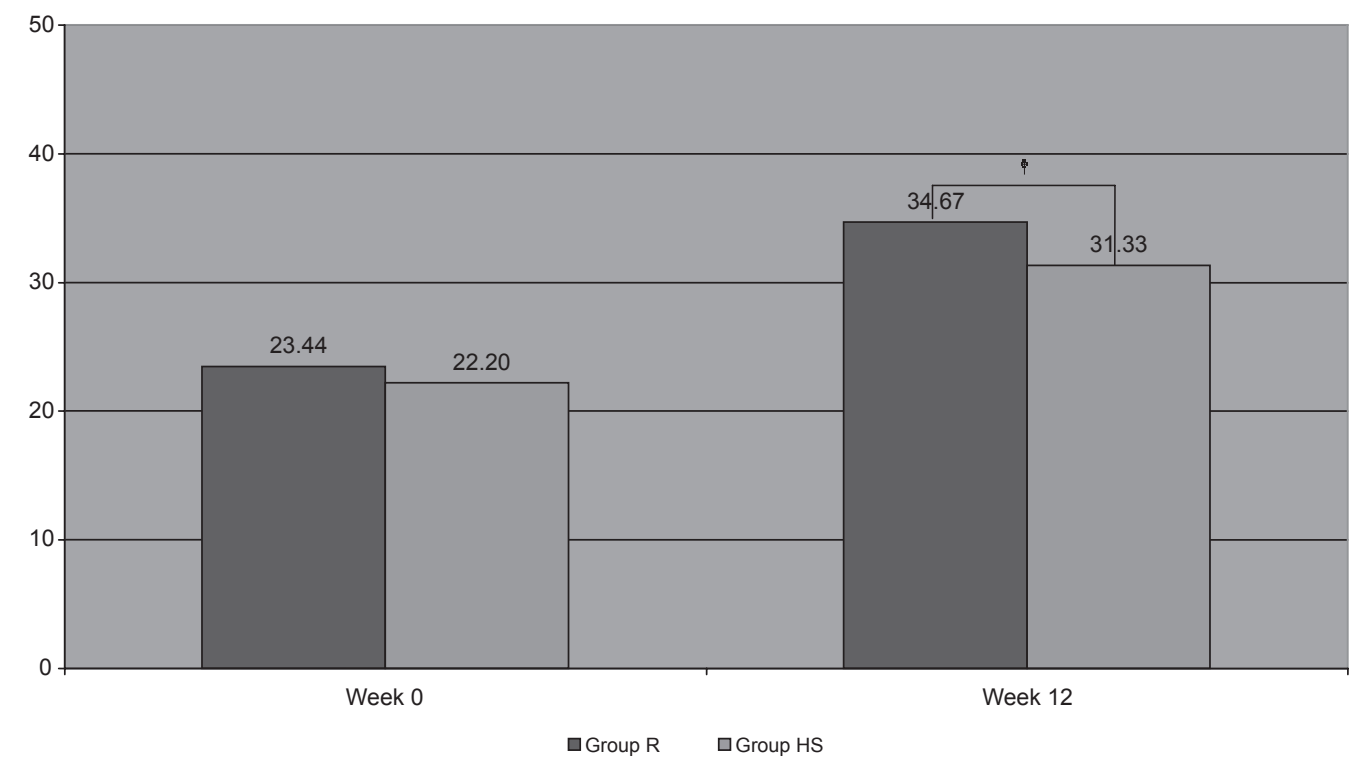

Figure 3 The grouped medians of Heinrich's Quality of Life Scale score at weeks 0 and 12.

Notes: ${ }^{\dagger} r_{m} \geq 0.3$. Wilcoxon test $(z)$ and effect size $\left(r_{m}\right)$ : Group R: week $0-12: z=-3.063 ; p<0.00 I ; r_{m}=0.68$. Group HS: week $0-12: z=-3.66 ; p<0.001 ; r_{m}=0.65$. Mann-Whitney $U$ test $(Z)$ and effect size $\left(r_{m}\right)$ : Group R vs Group HS: week 0:290.00; $Z=-0.881 ; p=0.384 ; r_{m}=0.12 ;$ week I2:66.50; $Z=-1.576 ; p=0.119 ; r_{m}=0.30$.

In Group HS there was an increase from -0.75 to 7.56 . The DAI score did not show any statistically significant difference and no effect size between the two groups.

\section{Patient Preference Scale}

At week 0, Group R patients assessed their previous medication more positively, compared to patients in Group HS. Similarly at week 12 , Group R patients showed higher preference for their study medication, compared to their previous medication. The rate of change of preference study algorithm, as compared to previous therapy, was comparable in both groups (+2 points).

\section{Duration of hospitalization}

There was no statistically significant difference and no effect size in the duration of hospitalization in all patients, who

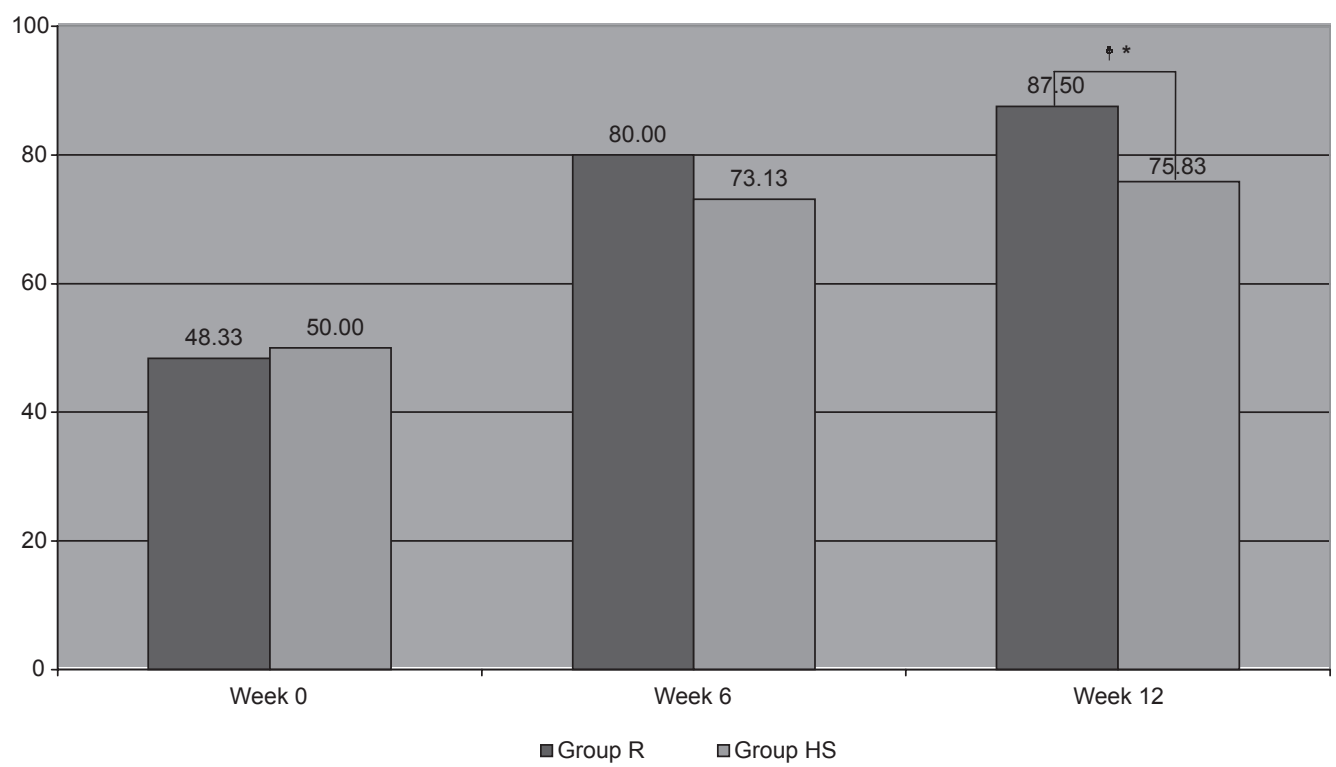

Figure 4 The grouped medians of Global Assessment of Functioning score at weeks 0,6, and I2.

Notes: ${ }^{*} p \leq 0.05 ;{ }^{\dagger} r_{m} \geq 0.3$. Wilcoxon test $(z)$ and effect size $\left(r_{m}\right)$ : Group R: week 0-6: $z=-3.523 ; p<0.001 ; r_{m}=0.65 ;$ week 0-12: $z=-3.068 ; p<0.001 ; r_{m}=0.68$. Group HS: week 0-6: $z=-4.026 ; p<0.00 I ; r_{m}=0.67$; week $0-12: z=-3.635 ; p<0.00 I ; r_{m}=0.67$. Mann-Whitney $U$ test $(Z)$ and effect size $\left(r_{m}\right)$ : Group R vs Group HS: week 0: $327.00 ; Z=-0.203 ; p=0.844 ; r_{m}=0.03 ;$ week $6: 142.00 ; Z=-1.343 ; p=0.183 ; r_{m}=0.22 ;$ week $12: 57.00 ; Z=-2.015 ; p=0.044 ; r_{m}=0.38$. 
completed the study (grouped medians: Group R: 39.00 days; Group HS: 40.00 days).

\section{Assessment of treatment safety}

Adverse events reporting

Spontaneous AEs were recorded throughout the study. The most frequently reported AEs were related to the extrapyramidal syndrome (EPS); sedation and blurred vision occurred rarely. A decrease in the number of AEs was noted during the study, especially in Group R.

\section{Simpson-Angus Extrapyramidal Symptoms \\ Rating Scale}

Extrapyramidal symptoms were rated with the SAS scale. Since they were mild in intensity, we simply recorded their presence or absence, without assigning a numerical score. At week 0, EPS in Group R occurred in $65.4 \%$ patients and in Group HS in $69.2 \%$ patients. These high EPS rates at baseline were due to the medications the patients were receiving previously as outpatients when we had no possibility to intervene. However, the intensity of the symptoms was very mild. At week 12, EPS occurred more frequently in Group HS, compared to Group R (52.9\% vs 33.3\%), but this difference did not reach statistical significance $(\mathrm{p}=0.324)$.

\section{Concomitant psychotropic medication}

There was high need for concomitant psychotropic medication in both Groups. In Group R, the medication most frequently used at week 1 was benzodiazepine anxiolytics (65.4\%) and nonbenzodiazepine hypnotics (50\%). At weeks 6 and 12 , the most frequent need was for nonbenzodiazepine hypnotics and antiparkinsonians, identically $50 \%$ for each group of drugs (Figure 5).

The most frequently indicated drugs in Group HS at weeks 1 and 12 were antiparkinsonians (80.8\%; 76.5\%). The administration of benzodiazepine anxiolytics (65.4\%) and non-benzodiazepine hypnotics (46.2\%) was comparable to Group R (Figure 6).

\section{Laboratory tests, vital signs, and body weight}

Comparison of laboratory tests from week 0 to week 12 did not reveal any significant differences. Blood pressure and pulse frequency were within normal limits throughout the study. No significant weight change was observed in any of the groups.

\section{Drop-out analysis}

Fourteen patients of Group R and 9 patients of Group HS dropped out of the study earlier, for different reasons (Table 3).

\section{Correlations between rating scales}

In both groups we observed a positive correlation between CGI-S score and negative subscore, the subscore of general psychopathology, and total PANSS score, thus the decrease of schizophrenic symptoms was accompanied by a decrease in

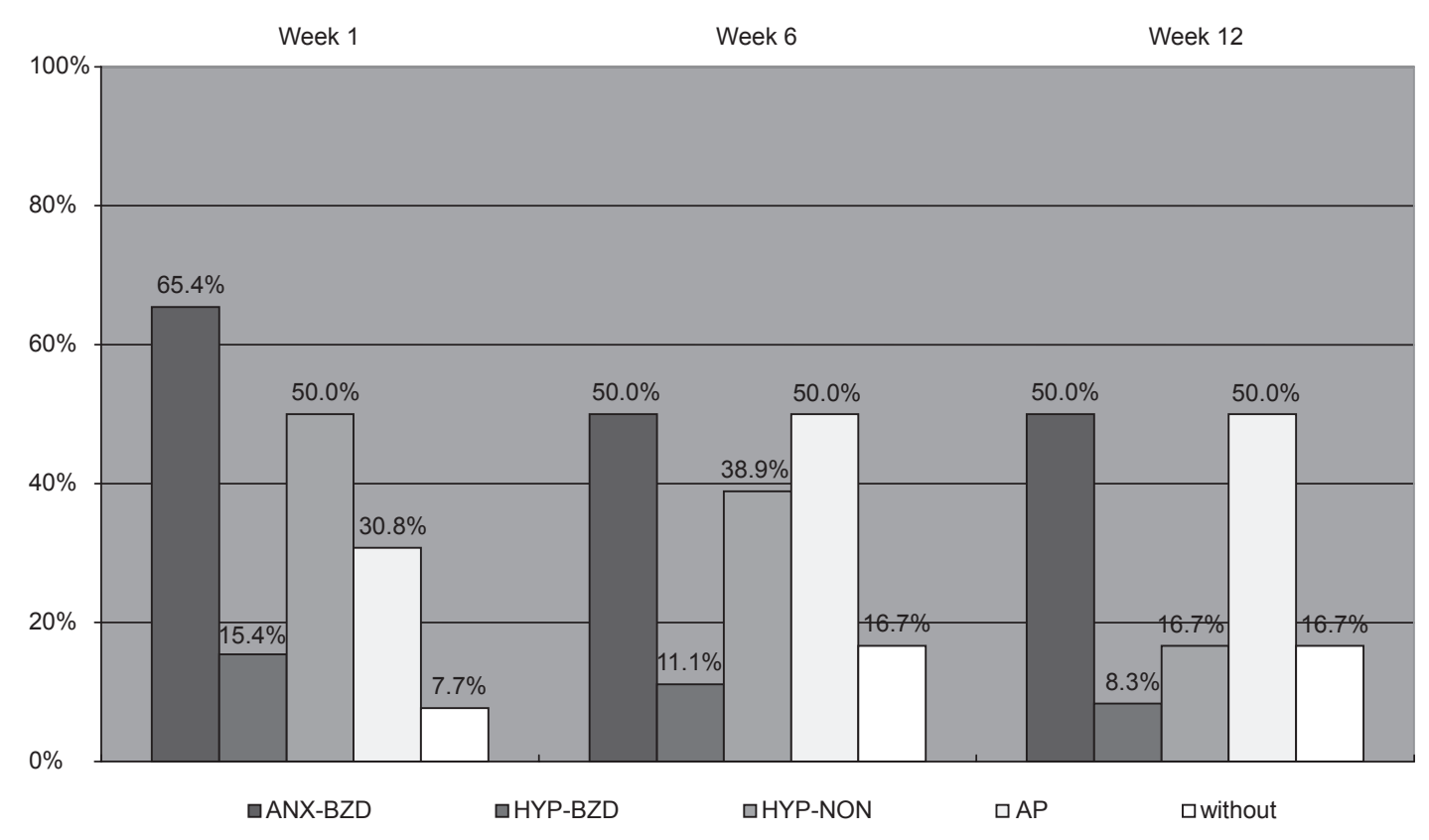

Figure 5 Concomitant psychotropic medication in Group R.

Abbreviations: ANX-BZD, benzodiazepine anxiolytics; HYP-BZD, benzodiazepine hypnotics; HYP-NON, nonbenzodiazepine hypnotics; AP, antiparkinsonians. 


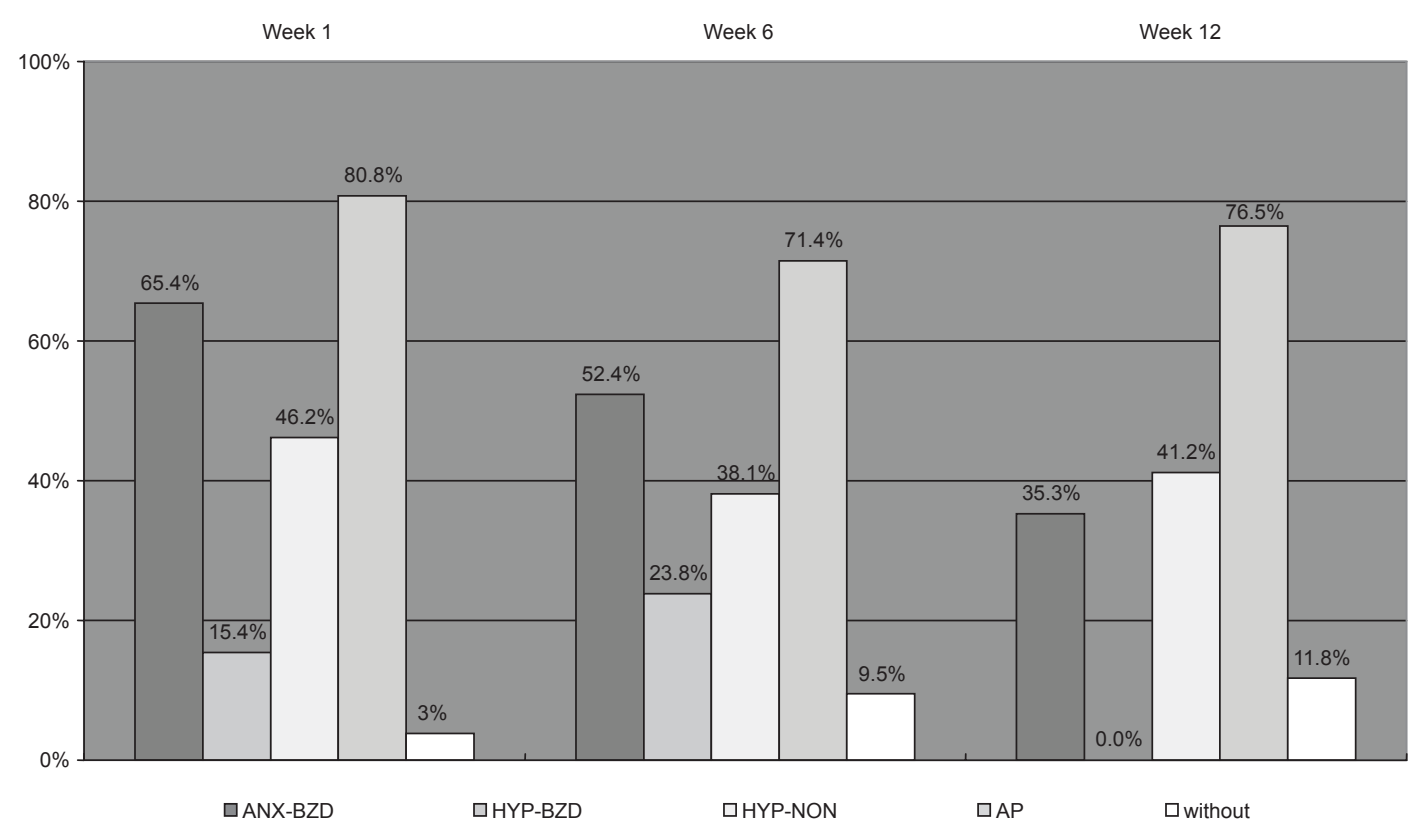

Figure 6 Concomitant psychotropic medication in Group HS.

Abbreviations: ANX-BZD, benzodiazepine anxiolytics; HYP-BZD, benzodiazepine hypnotics; HYP-NON, nonbenzodiazepine hypnotics; AP, antiparkinsonians.

disease severity. In Group R we found a negative correlation between CDSS and HQLS scores, ie, the decrease of schizophrenic symptoms was followed by an increase in patient's quality of life. This positive correlation between the negative PANSS subscore and quality of life was established in Group HS. In Group HS the remission of schizophrenic and depressive symptoms correlated with the improvement in disease severity, better subject attitude toward medication, netter quality of life and higher psychosocial functioning (Table 4).

\section{Discussion}

To the best of our knowledge, this is the first head to head comparison of the efficacy and safety of monotherapy with an atypical antipsychotic, risperidone, versus combination therapy using a typical antipsychotic, haloperidol, and a SSRI antidepressant, sertraline. Our results confirm that both therapeutic strategies are effective in the treatment of schizoaffective disorder, depressed type. However, when comparing the two treatments, we obtained statistically significant differences and a positive effect size favouring the efficacy and safety of risperidone monotherapy for the acute treatment of schizoaffective disorder, depressed type.

We confirmed our hypothesis that risperidone monotherapy would be more efficacious than combination therapy with haloperidol and sertraline for improving schizophrenic symptomatology and overall disease severity. However, we observed a comparable effect of both therapeutic regimens on negative schizophrenic and depressive symptoms and on their rate of global clinical improvement. Comparisons of the efficacy of haloperidol and risperidone in improving negative symptoms have appeared in several publications. ${ }^{23-26}$ Judging from those reports, the efficacy of risperidone versus haloperidol in patients with schizophrenic disorders with depressive symptoms is an unresolved question. In our study, the comparison included another factor, sertraline, an antidepressant extending the effect of haloperidol on affective symptoms, but concurrently represented a certain risk for potential drug interactions and possible worsening of schizophrenic symptoms. Müller-Siecheneder and colleagues ${ }^{27}$ compared the efficacy of haloperidol and amitriptyline therapy to the efficacy of risperidone in 123 patients with combination of psychotic and depressive symptoms in a six-week study. They observed a higher efficacy of combined therapy, although it was more frequently accompanied by AEs. The limitation of their study was the inclusion of patients with a combination of psychotic and depressive symptoms, and inclusion of psychotic depression.

With respect to quality of life and overall psychosocial functioning, our groups did not differ significantly at week 0 . Quality of life and overall functioning improved in both groups, however, more significantly in Group R. Despite this finding, we would have expected more robust differences between the groups. Tempier and Pawliuk ${ }^{28}$ in their retrospective cross-study analysis did not observe better quality of life in patients taking atypical antipsychotics than in patients treated with typical antipsychotics. These authors 
Table 2 Demographic and clinical characteristics of Groups R, HS and drop-outs R and HS

\begin{tabular}{|c|c|c|c|c|c|c|}
\hline \multirow[b]{2}{*}{ Group } & \multicolumn{2}{|l|}{ Week 0} & \multicolumn{2}{|l|}{ Week I2 } & \multicolumn{2}{|l|}{ Drop-outs } \\
\hline & $\mathbf{R}$ & HS & $\mathbf{R}$ & HS & $\mathbf{R}$ & HS \\
\hline Number (n) & 26 & 26 & 12 & 17 & 14 & 9 \\
\hline \multicolumn{7}{|l|}{ Age (years) } \\
\hline Mean & 41.92 & 44.92 & 42.92 & 44.94 & 41.07 & 44.89 \\
\hline SD & 13.08 & 10.40 & 11.48 & 10.92 & 14.68 & 9.98 \\
\hline Grouped median & 43.00 & 47.67 & 41.67 & 48.00 & 45.00 & 46.00 \\
\hline Range & $19-65$ & $20-61$ & $24-65$ & $20-61$ & $19-60$ & $29-57$ \\
\hline \multicolumn{7}{|l|}{ Living arrangement (n/\%) } \\
\hline Alone & $6 / 23.08 \%$ & $5 / 19.23 \%$ & $2 / 16.67 \%$ & $4 / 23.53 \%$ & $4 / 28.57 \%$ & $1 / I I . \mid I \%$ \\
\hline With partner & $20 / 76.92 \%$ & $21 / 80.77 \%$ & $10 / 83.33 \%$ & $13 / 76.47 \%$ & $10 / 71.43 \%$ & $8 / 88.89 \%$ \\
\hline \multicolumn{7}{|l|}{ Level of education (n/\%) } \\
\hline Elementary school & $3 / 11.54 \%$ & $2 / 7.69 \%$ & $1 / 8.33 \%$ & $2 / 11.76 \%$ & $2 / 14.29 \%$ & $0 / 0.00 \%$ \\
\hline High school without graduation & $4 / 15.38 \%$ & $7 / 26.92 \%$ & $1 / 8.33 \%$ & $5 / 29.41 \%$ & $3 / 21.43 \%$ & $2 / 22.22 \%$ \\
\hline High school with graduation & $1 \mathrm{l} / 42.31 \%$ & $9 / 34.62 \%$ & $4 / 33.33 \%$ & $5 / 29.41 \%$ & $7 / 50.00 \%$ & $4 / 44.45 \%$ \\
\hline Baccalaureate & $2 / 7.69 \%$ & $\mathrm{I} / 3.85 \%$ & $2 / 16.67 \%$ & $1 / 5.88 \%$ & $0 / 0.00 \%$ & $0 / 0.00 \%$ \\
\hline University & $6 / 23.08 \%$ & $7 / 26.92 \%$ & $4 / 33.33 \%$ & $4 / 23.53 \%$ & $2 / 14.29 \%$ & $3 / 33.33 \%$ \\
\hline \multicolumn{7}{|l|}{ Employment (n/\%) } \\
\hline Student & $1 / 3.85 \%$ & $1 / 3.85 \%$ & $\mathrm{I} / 8.33 \%$ & $1 / 8.33 \%$ & $0 / 0.00 \%$ & $0 / 0.00 \%$ \\
\hline Unemployed & $7 / 26.92 \%$ & $2 / 7.69 \%$ & $2 / 16.67 \%$ & $2 / 11.76 \%$ & $5 / 35.71 \%$ & $0 / 0.00 \%$ \\
\hline Employed & $6 / 23.08 \%$ & $6 / 23.08 \%$ & $4 / 33.33 \%$ & $4 / 23.53 \%$ & $2 / 14.29 \%$ & $2 / 22.22 \%$ \\
\hline Invalid pensioner & $10 / 38.46 \%$ & $14 / 53.85 \%$ & $4 / 33.33 \%$ & $8 / 47.06 \%$ & $6 / 42.86 \%$ & $6 / 66.67 \%$ \\
\hline Pensioner & $2 / 7.69 \%$ & $3 / 11.54 \%$ & $\mathrm{I} / 8.33 \%$ & $2 / 11.76 \%$ & $\mathrm{I} / 7.14 \%$ & I/II.II\% \\
\hline \multicolumn{7}{|l|}{ Duration of disorder (years) } \\
\hline Mean & 12.18 & 10.90 & 11.00 & 10.03 & 13.20 & 12.56 \\
\hline SD & 11.46 & 9.48 & 9.22 & 9.05 & 13.35 & 10.60 \\
\hline Grouped median & 8.33 & 7.50 & 9.00 & 6.20 & 6.00 & 10.00 \\
\hline Range & $0-39$ & $0.5-36$ & $0-26$ & $0.5-29$ & $0.25-39$ & $1-16$ \\
\hline \multicolumn{7}{|l|}{ Course of disorder (n/\%) } \\
\hline Episodic with progressive defect & $17 / 65.38 \%$ & $19 / 73.08 \%$ & $6 / 50.00 \%$ & $13 / 76.47 \%$ & $\mathrm{I} \mathrm{I} / 78.57 \%$ & $6 / 66.67 \%$ \\
\hline Episodic with remissions & $9 / 34.62 \%$ & $7 / 26.92 \%$ & $6 / 50.00 \%$ & $4 / 23.53 \%$ & $3 / 21.43 \%$ & $3 / 33.33 \%$ \\
\hline \multicolumn{7}{|c|}{ Polarity of schizoaffective disorder (n/\%) } \\
\hline Unipolar - depressed & $15 / 57.69 \%$ & $20 / 76.92 \%$ & $7 / 58.33 \%$ & $13 / 76.47 \%$ & $8 / 57.14 \%$ & $7 / 77.78 \%$ \\
\hline Bipolar - depressed & $11 / 42.31 \%$ & $6 / 23.08 \%$ & $5 / 41.67 \%$ & $4 / 23.53 \%$ & $6 / 42.86 \%$ & $2 / 22.22 \%$ \\
\hline
\end{tabular}

interpreted their results by assuming that higher demands are placed on the life of patients taking atypical antipsychotics than in those receiving typical antipsychotics.

In both groups we observed a comparable effect size and a positive change of patient attitude toward study medication (DAI scale). They not only had a better attitude toward study

Table 3 The reasons for drop-outs

\begin{tabular}{|c|c|c|c|c|c|c|c|c|}
\hline \multirow[b]{2}{*}{ Group } & \multicolumn{2}{|c|}{ Week 2} & \multicolumn{2}{|c|}{ Week 4} & \multicolumn{2}{|c|}{ Week 6} & \multicolumn{2}{|c|}{ Week 8} \\
\hline & $\mathbf{R}$ & HS & $\mathbf{R}$ & HS & $\mathbf{R}$ & HS & $\mathbf{R}$ & HS \\
\hline Number (n) & 6 & 4 & 2 & I & 4 & 4 & 2 & 0 \\
\hline \multicolumn{9}{|l|}{ Reason } \\
\hline Low efficacy & 4 & 3 & I & 0 & 1 & 2 & I & 0 \\
\hline Adverse events & I & 0 & I & 1 & 0 & 0 & 0 & 0 \\
\hline Mania & I & 1 & 0 & 0 & 0 & 0 & 0 & 0 \\
\hline Noncompliance & 0 & 0 & 0 & 0 & 3 & 2 & I & 0 \\
\hline
\end{tabular}

medication, as compared to their previous medication, but their attitude improved during the course of the trial. The positive change of attitude was particularly evident during the first six weeks but levelled off between week 6 and 12 in both groups. A likely explanation for this observation is that patients in an acute condition require relief during the first few weeks of treatment, and efficacious medication provides this relief. However, once the acute phase is over, the patient begins to consider the likely need for long-term therapy and becomes more concerned about the presence of adverse events. A more favorable assessment of the study medication in both groups, compared to the previous medication, was obtained with the PPS scale. In comparing the PPS scores at week 12 , Group R patients $(n=12)$ evaluated the study medication more favorably than patients in Group HS ( $n=17)$. This is in accord with our hypothesis and the results of published studies ${ }^{8,29}$ 
Table 4 Correlation analysis of scales

\begin{tabular}{|c|c|c|}
\hline \multicolumn{3}{|l|}{ Group $R(n=12)$} \\
\hline \multicolumn{2}{|l|}{ Dependent variables } & \multirow{3}{*}{$\begin{array}{l}\text { Spearman rank } \\
\text { correlation } \\
\text { coefficient "rho" }\end{array}$} \\
\hline & & \\
\hline PANSS negative subscore & CGI-S & \\
\hline PANSS general psychopathology & CGI-S & $0.739 * *$ \\
\hline PANSS total score & CGI-S & $0.717^{* *}$ \\
\hline CDSS & HQLS & $-0.696 *$ \\
\hline \multicolumn{3}{|l|}{ Group HS (n = I7) } \\
\hline Dependent variables & & $\begin{array}{l}\text { Spearman rank } \\
\text { correlation } \\
\text { coefficient "rho" }\end{array}$ \\
\hline PANSS negative subscore & HQLS & $-0.638 * *$ \\
\hline PANSS negative subscore & GAF & $-0.497^{*}$ \\
\hline PANSS negative subscore & CGI-S & $0.718 * *$ \\
\hline PANSS general psychopathology & CGI-S & $0.548^{*}$ \\
\hline PANSS general psychopathology & DAI & $-0.709 * *$ \\
\hline PANSS total score & CGI-S & $0.658^{* *}$ \\
\hline PANSS total score & GAF & -0.535 \\
\hline PANSS total score & DAI & $-0.584 *$ \\
\hline CDSS & DAI & $-0.544^{*}$ \\
\hline CDSS & CGI-S & $0.485^{*}$ \\
\hline CDSS & CGI-I & $0.519 *$ \\
\hline HQLS & CGI-S & $0.550^{*}$ \\
\hline GAF & DAI & $0.513^{*}$ \\
\hline
\end{tabular}

Notes: $* p<0.05 ; * * p<0.01$.

Abbreviations: CDSS, Calgary Depression Scale for Schizophrenia; CGI-S, Clinical Global Impression-Severity; DAI, Drug Attitude Inventory; GAF, Global Assessment of Functioning; HQLS, Heinrich's Quality of Life Scale; PANSS, Positive and Negative Syndromes Scale for Schizophrenia.

We did not validate our hypothesis that the mean duration of hospitalization would be shorter with risperidone therapy. Our results agree with the retrospective study of Flynn and colleagues $^{11}$ who failed to observe a relationship between duration of hospitalization and type of treatment for schizoaffective disorder, but who did establish a correlation between duration of illness and rate of improvement. With a longer history of schizoaffective disorder, the patient's condition stabilized faster and required a shorter hospitalization. We did not examine this relationship in the present study.

We assessed the safety of therapy by the incidence of AEs and the need for concomitant medication. Generally, the incidence of AEs was low with both treatment strategies. The AEs, which we observed using the SAS scale, and those spontaneously reported, belong primarily to the EPS spectrum. A decrease in the intensity of AEs was observed during the study, especially in Group R. Reduction of antipsychotic dose and administration of concomitant medication had a positive effect on AEs. The need for concomitant psychotropic medication was high in both Groups. The administration of concomitant medication at the beginning of study was justified by the need for symptom relief, such as insomnia, inner tension and anxiety. Generally higher doses of drugs are used at the beginning of antipsychotic therapy with treatment emergent AEs and the associated need for concomitant medication. Overall Group R showed lower need for concomitant medication than Group HS. No AEs were noted in vital signs, weight or laboratory measures in conjunction with either drug regimen.

The diagnosis of schizoaffective disorder, depressed type, has been the subject of many scientific discussions. The literature has been addressing it since its initial description and to the present day, as it is considered to be an ambiguous diagnostic entity. Many authors consider it to be a waste basket of diagnoses. In attempting to diagnose the disorder accurately, we applied ICD-10 and DSM-IV criteria. The diagnostic process comprised three independent phases. Firstly, the admitting psychiatrist made the diagnosis at the time of hospitalization. Secondly, the diagnosis had to be confirmed by the inpatient psychiatrist in charge of the patient while in the hospital. Thirdly, the psychiatric investigator confirmed the diagnosis and determined eligibility of the patient to be enrolled in the study based on the inclusion and exclusion criteria. Thus, we are satisfied that the patient cohort was diagnostically homogeneous.

The present study has a number of limitations. One of them is the somewhat low number of patients in the two groups. Due to our rigorous inclusion and exclusion criteria, the target population was relatively small. At the time the study was conducted, a total of 130 patients diagnosed with schizoaffective disorder, depressed type, were hospitalized. Of these, $41.5 \%$ met study criteria and signed informed consent. Schizoaffective disorder afflicts both sexes, however, it occurs far more frequently in women. The uneven sex distribution was supported by our study with predominant representation of female $(n=102)$ versus male $(n=28)$ patients. Ultimately, the entire study cohort analyzed consisted of female patients. Another limitation was the open design of the study reflecting a possible subjective bias. This weakness, however, was partially offset by the advantage of enabling the patient to work psychoeducationally with the treatment team and thereby improving the therapeutic relationship. In spite of these limitations, however, the data clearly established a significant therapeutic advantage of monotherapy with the atypical antipsychotic, risperidone.

\section{Conclusions}

The study demonstrated higher efficacy of risperidone monotherapy compared to combination therapy with haloperidol 
and sertraline, in regards to schizophrenic symptomatology, but suggested comparable efficacy in regards to depressive symptomatology for the acute treatment of schizoaffective disorder, depressed type. Risperidone monotherapy was more efficacious in improving quality of life and psychosocial functioning along with a more positive patient attitude toward therapy. Fewer adverse events and a lower need for concomitant medication in the risperidone group enhanced the patient's quality of life and decreased the pharmacoeconomic impact of therapy overall. For these reasons risperidone monotherapy may be advantageous for long-term administration. On the other hand, combination of haloperidol and sertraline represents a highly effective therapeutic alternative, especially suitable for patients who may not tolerate risperidone. A distinct advantage of the combination is the beneficial effect on depressive symptoms, which may represent an indication for such a regimen, if the affective component of this disorder predominates.

\section{Disclosure}

This manuscript was previously presented at the 20th ECNP Congress, October 13-17th, 2007, Vienna, Austria: Izáková L. Monotherapy and combination therapy of schizoaffective disorder, depressive type. Abstract in: Eur Neuropsychopharm. 2007;17(Suppl 4):s. 414. Dr. Halaris is registered with the Speaker's Bureau of Pfizer and Forest Laboratories and is a consultant for Pfizer.

\section{Acknowledgments}

Alojz Ritomský, PhD, Institute of Applied Psychology, Faculty of Social and Economic Sciences, Comenius University in Bratislava, Slovakia.

\section{References}

1. Lebrun T, Wilmotte J. Schizoaffective disorder: its nosographic significance. Acta Psychiatr Belg. 1993;93:220-235.

2. Lake CR, Hurwitz N. Schizoaffective disorders are psychotic mood disorders; there are no schizoaffective disorders. Psych Res. 2006;143:255-287.

3. Vollmer-Larsen A, Jacobsen TB, Hemmingsen R, Parnas J. Schizoaffective disorder - the reliability of its clinical diagnostic use. Acta Psychiatr Scand. 2006;113:402-407.

4. Kolibáš E, Čaplová T, Fleischer J, et al. Špeciálna psychiatria. Bratislava: Vydavatel'stvo UK; 1996:65.

5. Ebert MH, Loosen PT, Nurcombe B. Current Diagnosis and Treatment in Psychiatry, ed. USA: Lange Medical Books/McGraw Hill; 2000.

6. Lipkovich I, Deberdt W, Buckley PF, et al. Prediction of combined symptomatic and functional outcome in patients with schizophrenia and schizoaffective disorder. Neurotherapeutics. 2006;3:410.

7. Siris SG. Depression in schizophrenia: perspective in the area of "Atypical" antipsychotic agents. Am J Psychiatry. 2000;157:1379-1389.

8. Karow A, Schnedler D, Naber D. What would the patient choose? Subjective comparison of atypical and typical neuroleptics. Pharmacopsychiatry. 2006;39:47-51.
9. Clark RE, Bartels SJ, Mellman TA, Peacock WJ. Recent trends in antipsychotic combination therapy of schizophrenia and schizoaffective disorder: implications for state mental health policy. Schizophr Bull. 2002;28:75-84.

10. Turner MS, Stewart DW. Review of the evidence for the long term efficacy of atypical antipsychotic agents in the treatment of patients with schizophrenia and related psychoses. J Psychopharmacol. 2006;20:20-37.

11. Flynn J, Grieger TA, Benedek DM. Pharmacologic treatment of hospitalized patients with schizoaffective disorder. Psych Serv. 2002;53:94-96.

12. VojtekováL', Halaris A, Pospíšil R. Monoterapia versus kombinovaná liečba akútnej fázy depresívneho typu schizoafektívnej poruchy. Psych Pro Praxi. 2002;4:25-29.

13. Dóci I, Kovářová M, Kovaničová M. Liečba schizoafektívnej poruchy na Psychiatrickej klinike v Košiciach v roku 2002. Psychiatrie. 2003;2(Suppl):25-26.

14. Kay SR, Fiszbain A, Opler LA. The Positive and Negative Syndrome Scale (PANSS) for schizophrenia. Schizophr Bull. 1987;13:261-276.

15. Addington D, Addington J, Schissel B. A depression rating scale for schizophrenics. Schizophr Res. 1990;3:247-251.

16. Addington D, Addington J, Maticka-Tyndale E, Joyce J. Reliability and validity of a depression rating scale for schizophrenics. Schizophr Res. 1992;6:201-208.

17. Guy W. ECDEU Assessment for Psychopharmacology. Rockville: US DHEW; 1976. p. 534-537.

18. Heinrich DW, Hanlon TE, Carpenter WT Jr. The quality of life scale: an instrument for rating the schizophrenic deficit syndrome. Schizophr Bull. 1984;10:388-398.

19. Endicott J, Spitzer RL, Fleiss JL, Cohen J. The global assessment scale: A procedure for measuring the overall severity of psychiatric disturbance. Arch Gen Psychiatry. 1976;33:767-771.

20. Hogan TP, Awad AG, Eastwood R. A self-report scale predictive of drug compliance in schizophrenics: reliability and discriminative validity. Psychol Med. 1983;13:177-183.

21. Ross CK, Steward CA, Sinacore JM. The importance of patient preferences in the measurement of health care satisfaction. Med Care. 1993;31:1138-1149.

22. Simpson GM, Angus JWS. A rating scale for extrapyramidal side effects. Acta Psychiatr Scand. 1970;212(Suppl):11-19.

23. Janicak PG, Keck PE, Davis JM, et al. A double blind, randomized, prospective evaluation of the efficacy and safety of risperidone versus haloperidol in the treatment of schizoaffective disorder. J Clin Psychopharmacol. 2001;21:360-368.

24. Leucht S, Pitschel-Walz G, Abraham D, Kissling W. Efficacy and extrapyramidal side-effects of the new antipsychotics olanzapine, quetiapine, risperidone and sertindole compared to conventional antipsychotics and placebo: a meta-analysis of randomized controlled trials. Schizophr Res. 1999;35:51-68.

25. Marder SR, Meibach RC. Risperidone in the treatment of schizophrenia. Am J Psychiatry. 1994;151:825-835.

26. Hunter RH, Joy CB, Kennedy E, Gilbody SM, Song F. Risperidone versus typical antipsychotic medication for schizophrenia. Cochrane Database Syst Rev. 2003;2:CD000440.

27. Müller-Siecheneder F, Müller MJ, Hillert A, Szegedi A, Wetzel H, Benkert O. Risperidone versus haloperidol and amitriptyline in the treatment of patients with a combined psychotic and depressive syndrome. J Clin Psychopharmacol. 1998;18:111-120.

28. Tempier R, Pawliuk N. Influence of novel and conventional antipsychotic medication on subjective quality of life. J Psychiatr Neurosci. 2001;26:131-136.

29. Tiihonen J, Wahlbeck K, Lönnqvist J, Klaukka T, Ionnidis JPA, Volavka J, Haukka J. Effectiveness of antipsychotic treatments in a nationwide cohort of patients in community care after first hospitalisation due to schizophrenia and schizoaffective disorder: observational follow-up study. BMJ. 2006;333:224-229. 
